\title{
A CERENKOV RADIATION DETECTION SYSTEM FOR THE ADVANCED PHOTON SOURCE STORAGE RING*
}

\author{
A. Pietryla ${ }^{\dagger}$ and W. Berg, Argonne National Laboratory, Argonne, IL \\ R. Merl, Los Alamos National Laboratory, Los Alamos, NM
}

\begin{abstract}
A Cerenkov radiation detection system has been designed for Argonne National Laboratory's Advanced Photon Source 7-GeV electron storage ring. This system is intended for monitoring and measuring localized beam losses. The Cerenkov radiation detection system includes a detector assembly mounted at selected locations in the storage ring tunnel and an electronics package in a VME format. The electronics package consists of a high-voltage power supply, a 16-bit pulse discriminator-counter, two gated integrators, and two 16-bit analog-to-digital converters. The design of the timing circuit offers an extended range of $655 \mu$ s for both the gated integrator gate width and gate delay. The detectors have been installed near all storage ring scrapers, the storage ring thin-septum magnet, and at several insertion device locations. Plans are to instrument all present and future insertion devices. This paper discusses the electronics design as well as the mechanical design of the Cerenkov detector system.
\end{abstract}

\section{INTRODUCTION}

The Advanced Photon Source (APS) 1104-m storage ring is divided into 40 sectors. Potentially, 35 of the 40 sectors will have small-aperture (5-mm and $8-\mathrm{mm}$ ) chambers with insertion device (ID) sources installed. The potential for bremstrahlung radiation from particle losses at these points must be recognized and controlled. The Cerenkov radiation detection system is intended to measure these localized beam losses caused by missteering of the beam, and to aid in tuneup of the storage ring. This design is based on the beam loss monitor design used for the PEP-II $B$ Factory at the Stanford Linear Accelerator Center (SLAC) [1,2]. The detectors have been installed near each of the scrapers, the storage ring thinseptum magnet, and at a few of the small-aperture straight sections where the IDs are located.

Experimental Physics and Industrial Control System (EPICS) driver and database software were written to interface the design to our control system [3]. Motif Editor and Display Manager (MEDM) screens were also developed for an easy windowing interface to the hardware.

\footnotetext{
* Work supported by the U.S. Department of Energy, Office of Basic Energy Sciences under Contract No. W-13-109-ENG-38.

$\dagger$ afp@aps.anl.gov
}

\section{ELECTRONICS}

The electronics package for the Cerenkov radiation detection system includes a 16-bit pulse discriminatorcounter; two independent gated integrators, each with a 16-bit analog-to-digital converter (ADC) [4]; a timing circuit synchronous with the storage ring injection timing; an on-board high-voltage power supply; and a VME interface. The complete system also includes a photomultiplier tube (PMT) assembly installed at selected locations in the storage ring tunnel. A block diagram of this system is shown in Fig. 1.

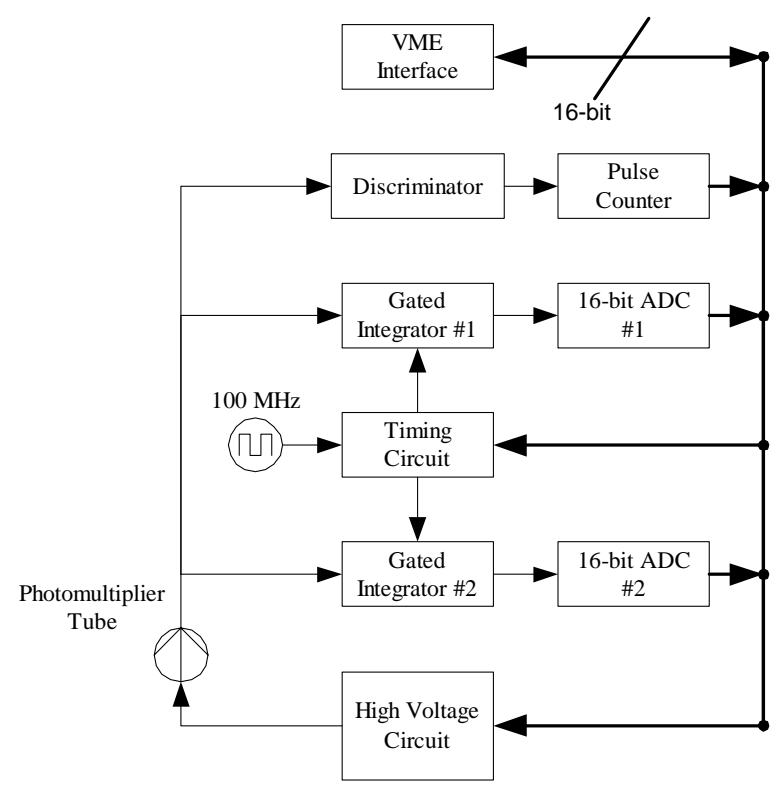

Figure 1: Cerenkov radiation detection system.

The pulse counter circuit discriminates the incoming pulses from the detector and compares them with a variable threshold. The threshold is set via EPICS. Any discriminated pulse with an amplitude greater than the threshold is passed to a 16-bit counter. This counter also features a programmable reset function, which allows counting for periods ranging from $153 \mu$ s up to $10 \mathrm{~s}$.

The gated integrators have different integration constants (75 ns and $250 \mathrm{~ns}$ ) to allow simultaneous measurements over various periods without saturating the integrators. Each integrator has independent gate width and delay controls programmable via EPICS. The range of control for the gate width is from $30 \mathrm{~ns}$ to $655 \mu \mathrm{s}$, while the delay range is from $110 \mathrm{~ns}$ to $655 \mu \mathrm{s}$. 
As mentioned earlier, the timing for the gated integrators in this system is synchronous with the injection trigger. This allows identification of the turn number for each measurement. This timing system, along with the wide range of delay, allows the observation and identification of any pulse up to the $178^{\text {th }}$ turn. Pulses from the detector can be integrated over a single turn or many turns. Because the timing of the two integrators is independent, the integrators can be set to measure the losses at different turns. This approach offers a great deal of flexibility for measurement configuration. The MEDM control screen example in Fig. 2 shows the "slow integrator" starting its integration period one turn (3.68 $\mu \mathrm{s})$ earlier than the "fast integrator." It also shows an integration period of 10 turns for both of the integrators.

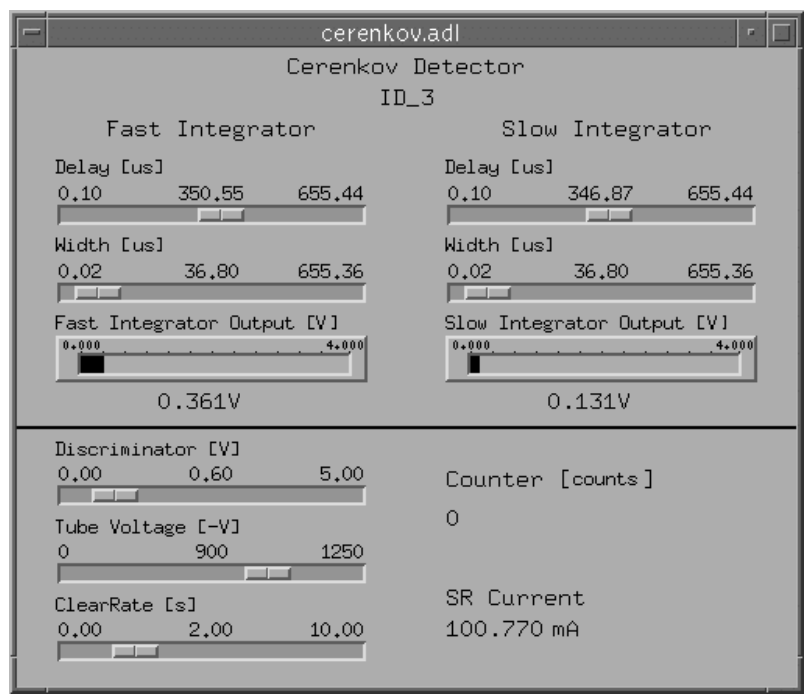

Figure 2: MEDM control screen for the Cerenkov detector at ID-3.

The high-voltage power supply for the PMT is a compact unit manufactured by Hamamatsu Corporation [5] and is an integral part of the VME board. It has a variable output of $0 \mathrm{~V}$ to $-1250 \mathrm{~V}$ and is programmable via EPICS.

\section{DETECTOR DESIGN}

The in-tunnel detector assembly is based on a design developed at the SLAC facility for the PEP-II $B$ factory $[1,2]$. The detector used for this system is a 16-mm PMT made by Hamamatsu [5]. It is housed in a corrosionresistant nickel-plated steel cylinder "can" filled with lead. Steel was chosen for the outer casing to provide shielding from fields generated by neighboring magnets in the storage ring. The lead liner is used to reduce background radiation at the PMT. The original SLAC design called for the cylinder to be poured with lead and then machined to accept the PMT and subcomponents. In our experience, this process made assembly of the detector unit difficult. A design was developed using lead that is machined to precisely fit into the shield housing, which gives us the flexibility to assemble the components externally and then insert the subassembly into the can.

A fused silica radiator couples the Cerenkov light to the faceplate of the PMT. Synthetic quartz was chosen as the substrate for the radiator and PMT window for its radiation resistance characteristics to avoid darkening of the optical materials. The radiator is aluminum coated on all sides except the exit port that faces the input of the PMT. A small amount of optical grease is used at the radiator and PMT faceplate interface to increase the coupling efficiency [6]. A force generated from a set of spring wave washers locates the radiator against the window. The mechanical design criterion is to firmly hold all the components in place so they will not shift or rattle once the unit is fully assembled.

The PMT tube socket was special ordered with radiation-resistant insulated leads rather than in the stock Teflon configuration. Because of the difficulty of locating high-voltage feedthroughs with radiation-resistant dielectrics in small quantities, a Teflon dielectric was selected as a low-cost alternative. For this reason, a shielding collar located outside the can was developed to protect the feedthroughs from radiation damage. To avoid ground loops, the PMT and associated components are electrically isolated from the shielding housing via a fiberglass spacer.

\section{PRELIMINARY RESULTS}

While this system is relatively new for the APS, some preliminary studies have been conducted. Some of the results are shown here, but a more in-depth discussion is presented in another publication in this conference [7].

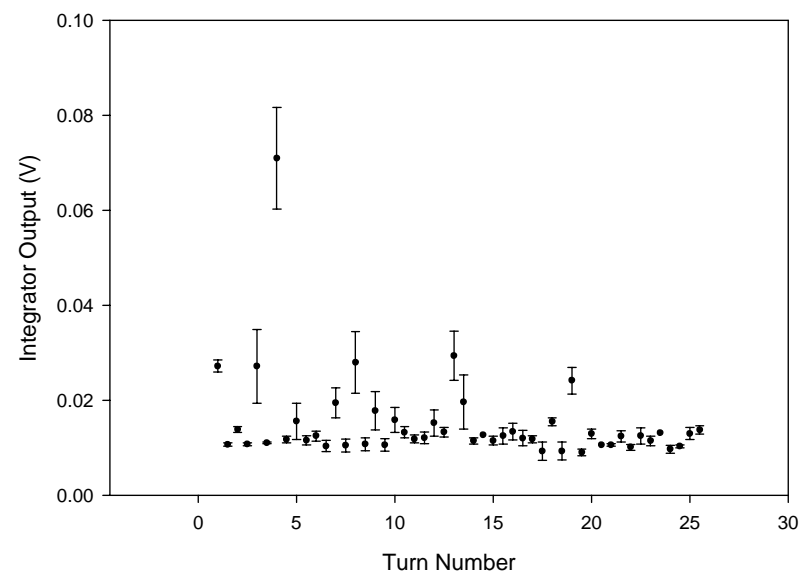

Figure 3: Beam losses versus turn number at the ID-3.

Using the system described in this paper, we have shown that the greatest losses do not necessarily occur on the first turn after injection. Figure 3 demonstrates that for ID-3, the largest loss occurs during the fourth turn. The data for this plot were obtained by collecting 10 samples per step with the integrator gate width to $1.8 \mu \mathrm{s}(<1 / 2$ turn $)$ and scanning the gate delay in half-revolution steps. Beam was continually injected into the storage ring at a $2-\mathrm{Hz}$ 
rate while the storage ring kicker magnets were set so that the beam made only one turn.

When just the detector output is considered, losses occur for many turns following injection (see Fig. 4). The data show the characteristics of both the injection betatron oscillation, seen in the first few tens of turns, as well as the synchrotron oscillation, seen in turn 80 and beyond. These data were collected using an oscilloscope during injection of a single bunch into the storage ring. The sector 39 horizontal scraper was positioned normally to define the momentum aperture for protecting downstream ID chambers in the event of a beam dump. However, in this case, the injection phase was not optimized and the bunch executed a quarter-phase oscillation in longitudinal-phase space and hit the scraper.

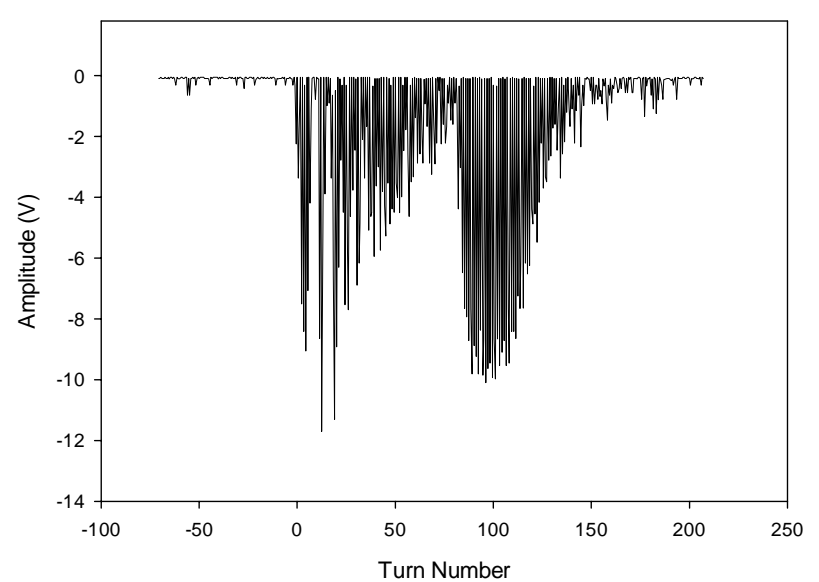

Figure 4: Output of the detector during an injection cycle. Data for this plot were collected at the detector located near the sector 39 horizontal scraper.

\section{CONCLUSION}

A Cerenkov radiation detection system has been designed for the Advanced Photon Source storage ring to sense and measure localized beam losses using an intunnel detector based on a SLAC PEP-II $B$ Factory design. New data acquisition electronics using a VME format have been developed. These include a 16-bit pulse discriminator-counter to measure losses in the presence of stored beam; two gated integrators, each with a dedicated 16-bit ADC to measure the magnitude of the losses during injection; and an on-board high-voltage power supply to bias the in-tunnel detector. The in-tunnel detector consists of a PMT mounted in a nickel-plated steel can with a removable lead lining.

This system was designed to be very flexible, allowing a wide range of configurations to cover any number of measurement scenarios. The timing circuit has an extended range so that measurements can be made on any beam revolution up to the $178^{\text {th }}$ turn. Measurements can also be made over many turns or any combination because the timing for each of the gated integrators is independent.

An EPICS driver and database were written to interface the electronics to our control system software. MEDM screens were also developed to easily control all adjustable parameters.

\section{ACKNOWLEDGMENTS}

The authors would like to thank Louis Emery for his insight into the details of the data and his assistance with commissioning of this system. We also want to thank Glenn Decker for his enthusiastic support of this project.

\section{REFERENCES}

[1] A. Fischer et al., "Diagnostics Development for the PEP-II $B$ Factory," AIP Conference Proceedings 390, Beam Instrumentation Workshop, Argonne, IL, pp. 248-256 (1996).

[2] A. Fisher, "Instrumentation and Diagnostics for PEPII," AIP Conference Proceedings 451, Beam Instrumentation Workshop, Stanford, CA, pp. 107 (1998).

[3] http://www.aps.anl.gov/epics

[4] X. Wang, "Ultrafast, High Precision Gated Integrator," AIP Conference Proceedings 333, American Institute of Physics, New York, pp. 260266 (1994).

[5] Hamamatsu Corporation, Bridgewater, NJ.

[6] Bicron Corporation, Newbury, $\mathrm{OH}$.

[7] L. Emery and A. Pietryla, "Use of Cerenkov Radiation Detectors in Detecting Beam Losses in the Advanced Photon Source Storage Ring," Proc. Of PAC 2001. 